J

\title{
Posttraumatic epilepsy and treatment
}

\author{
James W. Y. Chen, MD, PhD; ${ }^{1-2 *}$ Robert L. Ruff, MD, PhD; ${ }^{3-4}$ Roland Eavey, BA; ${ }^{1-2}$ Claude G. Wasterlain, MD $^{1-2}$ \\ ${ }^{1}$ Department of Veterans Affairs (VA) Greater Los Angeles Healthcare System, Los Angeles, CA; ${ }^{2}$ Department of \\ Neurology, David Geffen School of Medicine at University of California, Los Angeles, Los Angeles, CA; ${ }^{3}$ Neurology \\ Service, Louis Stokes Cleveland VA Medical Center, Cleveland, $\mathrm{OH} ;{ }^{4}$ Case Western Reserve University School of \\ Medicine, Cleveland, $\mathrm{OH}$
}

\begin{abstract}
Posttraumatic epilepsy (PTE) is a major long-term complication of traumatic brain injury (TBI). PTE usually develops within 5 years of head injury. The risk for developing PTE varies with TBI type. Both Korean and Vietnam war veterans with penetrating TBI had a 53\% risk of developing PTE. The risk of developing PTE is between 10\% and 25\% in combat-associated closed-head trauma with positive brain imaging and about $5 \%$ in moderately severe closed-head injury without imaging finding. We do not know the risk of PTE among Operation Iraqi Freedom/Operation Enduring Freedom veterans with minimal TBI because of blast exposure. Partial seizures may manifest with subtle behavioral alterations that can be mistaken for manifestations of posttraumatic stress disorder and improperly treated. Accidents and medical complications commonly occur during seizures. Sudden unexpected death in epilepsy is most frequent among 20- to 40-year-olds. Seizures increase the likelihood of refractory seizures years after TBI. Seizures are also a social stigma that compromise veterans' reintegration into society. People with uncontrolled epilepsy are not allowed to drive and have difficulty obtaining or maintaining employment. Optimal seizure control is essential to the physical and emotional health of veterans with TBI and to their ability to lead productive lives.
\end{abstract}

Key words: anticonvulsants, brain imaging, posttraumatic epilepsy, posttraumatic stress disorder, refractory seizures, rehabilitation, seizures, traumatic brain injury, unprovoked seizures, veterans.

\section{INTRODUCTION}

\section{Definition of Posttraumatic Epilepsy}

The working definition of posttraumatic epilepsy (PTE), based on the traditional definition of epilepsy, is two or more unprovoked seizures after a head injury. Unprovoked seizures are defined as those that occur more than a week after the head injury. However, in one population-based study, 86 percent of patients with one unprovoked posttraumatic seizure experienced a second seizure within 2 years [1]. This observation suggests that

\footnotetext{
Abbreviations: AED $=$ antiepileptic drug, $\mathrm{APOE}=$ apolipoprotein $\mathrm{E}, \mathrm{CBZ}=$ carbamazepine, $\mathrm{CPSE}=$ complex partial status epilepticus, $\mathrm{CT}=$ computed tomography, DOD $=$ Department of Defense, EEG = electroencephalography, EMU = epilepsy monitoring unit, GABA = gamma-aminobutyric acid, LOC = loss of consciousness, MRI $=$ magnetic resonance imaging, OIF/OEF = Operation Iraqi Freedom/Operation Enduring Freedom, $\mathrm{PB}=$ phenobarbital, $\mathrm{PET}=$ positron emission tomography, $\mathrm{PHT}=$ phenytoin, $\mathrm{PTE}=$ posttraumatic epilepsy, $\mathrm{PTSD}=$ posttraumatic stress disorder, TBI = traumatic brain injury, VA = Department of Veterans Affairs, VHA = Veterans Health Administration, VISN = Veterans Integrated Service Network, VPA = valproic acid.

${ }^{*}$ Address all correspondence to James W. Y. Chen, MD, PhD; West Los Angeles VA Healthcare Center, Neurology (MC 127), 11301 Wilshire Blvd, Los Angeles, CA 90073; 310-268-3017; fax: 310-268-4611. Email: jwychen@ucla.edu DOI:10.1682/JRRD.2008.09.0130
} 
the risk of developing PTE in patients with one unprovoked posttraumatic seizure is extremely high. Consequently, many published articles define PTE as one or more unprovoked seizures late after head injury. This definition of PTE has the advantage of allowing anticonvulsant treatment (antiepileptic drugs [AEDs]) to begin early instead of waiting an unspecified period of time for a second seizure to occur.

Seizures that occur within the first 7 days after traumatic brain injury (TBI) are defined as provoked seizures. The pathogeneses, clinical presentations, and longterm outcomes of provoked and unprovoked seizures may differ. For instance, we know that treatments with AEDs are effective in reducing the risk of provoked seizures but not unprovoked seizures. Clinical trials of PTE must assess these two types of seizures separately.

\section{Risk of Posttraumatic Epilepsy After Traumatic Brain Injury}

The probability of PTE significantly correlates with the severity of injury. In general, TBI may be divided into three groups according to the severity of injury: mild (loss of consciousness [LOC], posttraumatic amnesia for $<30 \mathrm{~min}$, and no skull fracture), moderate (LOC, posttraumatic amnesia lasting $30 \mathrm{~min}$ to $24 \mathrm{~h}$, or skull fracture), and severe (brain contusion or intracranial hematoma, LOC, or posttraumatic amnesia $>24 \mathrm{~h}$ ). In a population-based study, the 5-year cumulative probability of seizures was 0.7 percent in patients with mild TBI, 1.2 percent with moderate TBI, and 10.0 percent with severe TBI [2]. The 30-year cumulative incidence was 16.7 percent. In a different study, approximately twothirds of patients experienced at least one seizure within 1 year and approximately 80 percent within 2 years [3]. The incidence of PTE is highest after wartime penetrating head injuries, presumably because of their severity [4]. The Figure compares the cumulative probability from different studies of late unprovoked seizures after TBI. One caveat of interpreting these clinical studies is that they only include clinical seizures. This approach could significantly underestimate the number of epileptic events and incidence of PTE by omitting the electrographic seizures.

Multivariate analysis showed that depressed skull fracture, brain contusion, intracranial hemorrhage, coma duration, low Glasgow Coma Scale score, and older age are the high-risk factors for PTE. The presence of early seizures did not increase the risk of PTE in patients with mild TBI.
Modern neuroimaging methods such as magnetic resonance imaging (MRI), positron emission tomography (PET) scan, or diffusion tensor imaging, which visualize the brain, are far superior to simple skull X-rays in detecting traumatic brain hemorrhage, axonal injury, and other types of TBI but have not yet been used in large long-term prognostic studies for identifying risk factors.

\section{Risk of Posttraumatic Epilepsy in Veterans with Traumatic Brain Injury}

The rates of PTE in veterans with combat-related TBI in World War I, World War II, and the Korean war ranged from 35 to 45 percent [5]. In the Vietnam Head Injury Study, 53 percent of veterans with penetrating head injury developed at least one seizure [4]. In the Korean war, no treatment was provided to veterans, while in the Vietnam war, phenytoin (PHT) was given for 6 months to all veterans with penetrating TBI without any effort to monitor compliance. This treatment did not reduce the incidence of PTE, although the type of injury differed (mostly shrapnel wounds in the Vietnam war and bullet wounds in the Korean war) and survival after severe injuries was much higher in the Vietnam war. In other words, the TBIs in the Vietnam war may have been more severe, leaving the basis for the higher likelihood of seizures after penetrating TBI for Vietnam war veterans unresolved. In about 50 percent of patients, the first seizure occurred within 1 year of TBI, but about 15 percent of patients developed PTE more than 5 years after TBI. The risk factors for developing PTE after penetrating head injury include retained metal fragments, intracranial hemorrhage, chronic neurological deficits, and brain parenchyma loss.

\section{Natural History of Posttraumatic Epilepsy}

PTE presents with different seizure frequency and may evolve into remission or develop into intractable seizures. The lifetime total number of seizures after TBI varies among patients. In the Korean war study, 39 percent of the veterans had 1 to 3 seizures during a 10-year follow-up period, while 38 percent had $>30$ seizures [6]. The seizure remission rates in PTE were about 25 to 40 percent in nonpenetrating head injury [7]. Frequent seizures in the first year after penetrating head injury suggest a reduced chance of remission. An estimated 13 percent of PTE patients become refractory to AED therapy [8]. 


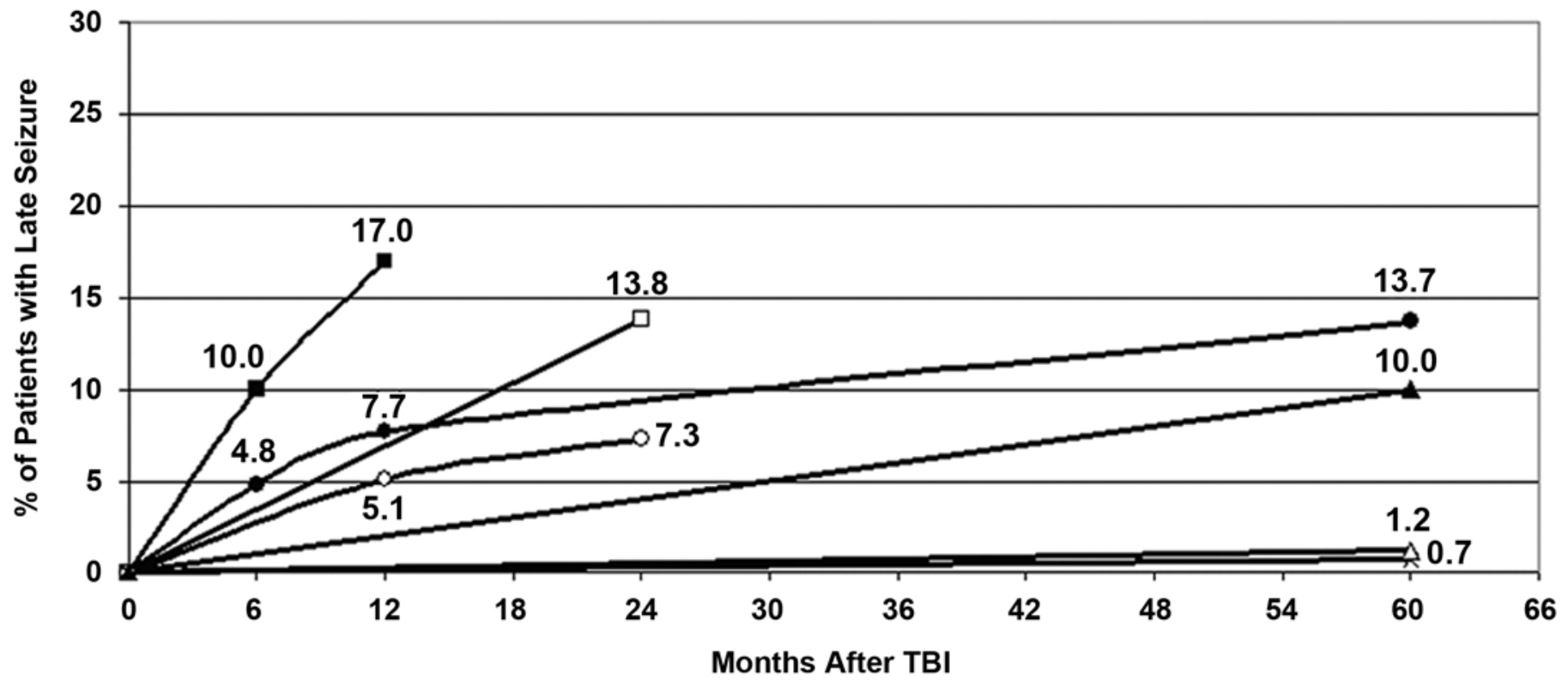

Figure.

Cumulative probability of late unprovoked seizures after traumatic brain injury (TBI) in seven studies. Mild to moderate TBI is correlated with lower risk of developing posttraumatic epilepsy (PTE). Prevalence of PTE was 10 to 17 percent with maximal follow-up period of 5 years. peds = pediatrics. [1] Asikainen I, Kaste M, Sarna S. Early and late posttraumatic seizures in traumatic brain injury rehabilitation patients: Brain injury factors causing late seizures and influence of seizures on long-term outcome. Epilepsia. 1999;40(5):584-89. [PMID: 10386527]. [2] Englander J, Bushnik T, Duong TT, Cifu DX, Zafonte R, Wright J, Hughes R, Bergman W. Analyzing risk factors for late posttraumatic seizures: A prospective, multicenter investigation. Arch Phys Med Rehabil. 2003;84(3):365-73. [PMID: 12638104]; DOI:10.1053/apmr.2003.50022. [3] Annegers JF, Hauser WA, Coan SP, Rocca WA. A population-based study of seizures after traumatic brain injuries. N Engl J Med. 1998;338(1):20-24. [PMID: 9414327]; DOI:10.1056/NEJM199801013380104. [4] Angeleri F, Majkowski J, Cacchiò G, Sobieszek A, D’Acunto S, Gesuita R, Bachleda A, Polonara G, Królicki L, Signorino M, Salvolini U. Posttraumatic epilepsy risk factors: One-year prospective study after head injury. Epilepsia. 1999; 40(9):1222-30. [PMID: 10487184]; DOI:10.1111/j.1528-1157.1999.tb00850.x. [5] Jennett WB, Lewin W. Traumatic epilepsy after closed head injuries. J Neurol Neurosurg Psychiatry. 1960;23:295-301. [PMID: 13789853]; DOI:10.1136/jnnp.23.4.295

\section{POSTTRAUMATIC EPILEPSY IN CONTEXT OF OIF/OEF}

\section{Medical Cost of Posttraumatic Epilepsy}

Epilepsy is a major long-term complication of TBI. PTE usually develops after a seizure-free interval. Most cases occur within 5 years of TBI, but the literature has described latent periods of up to 20 years. Consequently, PTE may not be present during initial TBI rehabilitation. Poorly controlled epilepsy produces medical and social complications. Uncontrolled seizures can be fatal. The abnormal brain electrical activity that occurs during a seizure may alter the properties of interneuronal connections, which increases the likelihood of future seizures. Thus, seizures may beget seizures. Veterans can aspirate during seizures, which can lead to pneumonia and damage to the airways and lungs. Generalized seizures disrupt blood pressure and heart rate control, which can lead to sudden death. Seizures also disrupt respiratory and body temperature control. Uncontrolled seizure activity can injure a patient through trauma, hyperthermia, or hypoxemia.

\section{Socioeconomic Cost of Posttraumatic Epilepsy}

The social consequences of epilepsy are enormous. Seizures are a social stigma that compromise veterans' reintegration into society. People with uncontrolled epilepsy are 
unable to hold an automotive, marine, or aviation operator's license. They have difficulty obtaining or maintaining employment. Accidents (e.g., drowning, burning, aspiration, fractures) and medical complications (e.g., pneumonia, hypertension, hypoxia, cardiac arrhythmias) commonly occur during seizures. Sudden unexpected death in epilepsy is most frequent among 20- to 40-year-olds. Partial seizures may manifest with subtle behavioral alterations that clinicians can mistake for mood alterations associated with conditions such as posttraumatic stress disorder (PTSD). If the abnormal behavior is not recognized as a manifestation of epilepsy, it may be improperly treated. Seizures alter interneuronal connections and increase the likelihood of more seizures. Uncontrolled seizures may become refractory to medication years after TBI. Optimal seizure control is essential for the physical and emotional health of veterans with TBI.

\section{Risk of Posttraumatic Epilepsy in OIF/OEF Veterans with Traumatic Brain Injury}

The risk for developing PTE varies depending on the type of TBI. Based on data from the Vietnam war, veterans with penetrating brain injuries have a 53 percent risk of developing PTE [4]. Among veterans with combat-associated closed-head trauma, the likelihood of developing PTE relates to the presence of cerebral injury that can be seen on cerebral imaging such as computed tomography (CT) or MRI scans. If cerebral injury can be seen on clinical imaging, the risk of developing PTE is between 10 and 25 percent. The presence of intracranial hemorrhage increases the risk of PTE. The likelihood of developing epilepsy for nonpenetrating moderately severe head injury without damage seen on CT or MRI is about 5 percent. The risk of PTE among veterans with minimal TBI because of blast exposure is unknown. However, if the risk is similar to that associated with minimal TBI produced by percussive head injury, a 1 percent risk of PTE exists among veterans with minimal TBI resulting from blast exposure. However, it is quite likely that more energy is conveyed to the brain tissue during the blast-injury impact. The estimated 1 percent risk could be at the lower margin. The potential risk of developing PTE might be as high as 5 percent, which is estimated from the nonpenetrating, moderately severe TBI cases. Whether repeated events of minimal TBI increase the risk of PTE is unknown.

\section{Nonconvulsive Seizures}

As mentioned earlier in this article, partial seizure, such as complex partial seizure, may manifest with subtle behavioral alterations that can be mistaken for apathy, catatonia, or mood alterations associated with conditions such as PTSD. The presentation of a complex partial seizure could be featureless to the untrained eye and might elude detection by a clinician who is unfamiliar with this condition. For instance, the patient could just be sitting or standing still with both eyes open and/or showing minor automatism of the hand or mouth for several seconds to a few minutes. In addition, an untreated patient might develop nonconvulsive complex partial status epilepticus (CPSE), which is a persistent epileptic state but with partially reserved brain function. The first reported case of CPSE occurred in 1887 with a patient who found himself 18 miles from the center of Paris, France, in a town called Claye that he had never visited before. Hughlings Jackson describes a famous case of CPSE: a medical doctor he called Dr. Z who suffered attacks of prolonged complex partial seizures [9]. Dr. Z could often continue the task at hand during these seizures. In one event, he went into a nonconvulsive seizure while examining a patient. When he finally awoke from the seizure, he had a different patient in front of him in his office. He has no memory of the time between. Dr. Z had completed the medical record of the previous patient with a correct diagnosis ("pneumonia of the left base") and had placed the patient on immediate bed rest, actions of which Dr. Z had no recollection at all.

For early detection of nonconvulsive seizure in patients with TBI, it is important that every clinician who treats patients with TBI is aware of this condition. We recommend further workup by a neurologist or epileptologist if a patient with TBI presents with a clinical history that suggests a nonconvulsive seizure. Capturing these events on electroencephalography (EEG) with video recording is often needed to establish a diagnosis of nonconvulsive seizure.

Table 1 indicates the number of Operation Iraqi Freedom/Operation Enduring Freedom (OIF/OEF) veterans with moderate or severe TBI as recognized by the Department of Defense (DOD). It includes neither veterans with TBI that the Veterans Health Administration (VHA) identified by screening OIF/OEF veterans for TBI nor military personnel who suffered TBI and remain with the DOD or have not been treated by the VHA. As of April 2007, the VHA had treated 446 veterans with penetrating brain injury and 11,703 OIF/OEF veterans with closed-head injury that produced moderate or severe TBI.

Table 2 indicates the estimated number of PTE cases among the 1.5 million military personnel who have 
already served in OIF/OEF. It includes the cases of TBI shown in Table 1 that the DOD recognized before the veterans received care through the VHA. Table 2 also considers the military personnel who served in OIF/OEF and have not yet been treated by the VHA. At the August 2007 Polytrauma Support Clinic Team meeting, Dr. Barbara Sigford, National Program Director for the VA Department of Physical Medicine and Rehabilitation, presented data on OIF/OEF. She indicated that there have been 2.2 million individual deployments to OIF/OEF by 1.5 million unique individuals. Comparing Table 1 with the data presented by Dr. Sigford suggests that approximately 1.25 million military personnel who served in OIF/OEF had not entered VHA care as of April 2007. The 1.25 million people include individuals who remain in DOD, some of who are receiving medical treatment through DOD. If one assumes that the fraction of individu- als with moderate or severe TBI among those who have not yet entered VHA care is 50 percent of that seen among individuals who have entered VHA care, then an additional 3,517 individuals with moderate/severe or penetrating TBI may enter VHA care (Table 2). As of December 2007, the Defense and Veterans Brain Injury Center reported 4,127 veterans wounded with TBI. The most recent report to Congress in March 2008 by the Defense Veterans Brain Injury Center identified a little over 32,000 injuries and around 5,500 individuals with TBI.

A recent survey from the RAND Corporation found that 19 percent of servicemembers $(n=320,000)$ reported experiencing a possible TBI while deployed, but only 43 percent of these received a physician evaluation. Among the factors accounting for the lack of medical attention was the servicemembers' fear of damaging their careers [10].

Table 1.

Operation Iraqi Freedom/Operation Enduring Freedom (OIF/OEF) veterans with moderate to severe traumatic brain injury (TBI) as recognized by Department of Defense.

\begin{tabular}{lr}
\hline \multicolumn{1}{c}{ Characteristic of TBI } & OIF/OEF Veterans* $^{*}$ \\
TBI Blast Injury (more than minimal, nonpenetrating-diagnosed in field) & 6,398 \\
TBI Penetrating (bullet, fragment, or other missile injury) & 446 \\
TBI Fall (more than minimal) & 2,795 \\
TBI Vehicular (more than minimal) & 2,510 \\
Moderate Closed-Head TBI with Penetrating Brain Injury & 11,703 \\
Total TBI (veterans with confirmed TBI not just symptoms) & 23,852 \\
\hline
\end{tabular}

*As of April 2007, 243,945 OIF/OEF veterans were receiving care from Department of Veterans Affairs.

Table 2.

Estimated numbers of veterans who will develop posttraumatic epilepsy (PTE) based on number of soldiers who have already been deployed in Operation Iraqi Freedom/Operation Enduring Freedom (OIF/OEF).

\begin{tabular}{|c|c|c|c|}
\hline Category & No. OIF/OEF Veterans & $\begin{array}{c}\% \text { at Risk } \\
\text { of PTE }\end{array}$ & $\begin{array}{c}\text { Estimated No. } \\
\text { of PTE }\end{array}$ \\
\hline \multicolumn{4}{|l|}{ As Recognized by DOD } \\
\hline Penetrating TBI & 446 & 53 & 237 \\
\hline Moderate Closed-Head TBI & 11,703 & 10 & 1,171 \\
\hline \multicolumn{4}{|l|}{ 1.25 Million Not Yet in VHA ${ }^{*}$} \\
\hline Penetrating TBI & 1,115 & 53 & 591 \\
\hline Moderate Closed-Head TBI & 29,258 & 10 & 2,926 \\
\hline \multicolumn{4}{|l|}{ 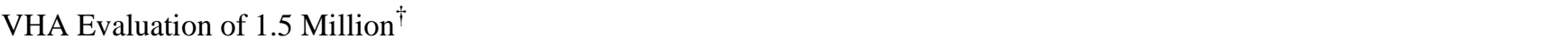 } \\
\hline Mild TBI from VHA Screen & 300,000 & 1 & 3,000 \\
\hline Total Estimated No. of PTE & - & - & 7,925 \\
\hline \multicolumn{4}{|c|}{$\begin{array}{l}\text { *Assumes that incidence of TBI among individuals who served in OIF/OEF and have not entered VHA is 50\% of that seen among OIF/OEF veterans who are being } \\
\text { treated by VHA. } \\
{ }^{\dagger} \text { Assumes that } 20 \% \text { of those who served in OIF/OEF have minimal TBI. }\end{array}$} \\
\hline
\end{tabular}


While not all OIF/OEF veterans will seek VHA care, most veterans with PTE will likely seek VHA treatment for the following reasons:

- Epilepsy will likely prevent them from working, leading to a loss of employment that can result in loss of private-sector healthcare coverage.

- A veteran with seizures may seek treatment through the VHA to be evaluated by the Veterans Benefits Administration (VBA) for benefits.

Table 2 also includes the estimated number of veterans with minimal TBI who have been or will be identified as having TBI through the VHA screening process. To date, more than 20 percent of OIF/OEF veterans who have not already been identified by DOD as having TBI will screen positive on the TBI screening questionnaire. The experience in Veterans Integrated Service Network (VISN) 10, for example, has been that clinicians find most of the individuals who screen positive on the questionnaire to have at least minimal TBI after further medical and neuropsychological assessment. Additionally, after clinicians completed medical and neuropsychological assessments, VISN 10 found that more than 20 percent of OIF/OEF veterans had at least minimal TBI. If the experience in VISN 10 holds true for the remainder of the VISNs, then the number of individuals who have served in OIF/OEF and have at least minimal TBI would be about 300,000 individuals $(0.2 \times 1.5$ million). The estimate of 300,000 cases of minimal or more severe TBI does not include future deployments. Thus, based on current deployments, Table 2 shows that about 7,900 cases of PTE resulting from OIF/OEF will exist. The number of PTE cases will increase if hostilities continue.

About 9,600 veterans who did not serve in OIF/OEF are treated by the VHA for epilepsy. The addition of the estimated 7,924 veterans who will develop PTE in association with OIF/OEF service will increase the VHA epilepsy population by 83 percent and might significantly strain the care for epilepsy patients within the VHA. Furthermore, the estimated number of 9,600 veterans who are currently treated within VHA for epilepsy might not reflect the real needs for epilepsy care within the VHA. The number of total enrollees in the Department of Veterans Affairs (VA) Care System in fiscal year 2007 was 7.8 million. Assuming the same epilepsy prevalence rate of approximately 1 percent translates to about 78,000 veterans who might need epilepsy care within the VHA.

\section{POSTTRAUMATIC EPILEPSY TREATMENT}

\section{Introduction}

Epilepsy treatment has advanced greatly since the Vietnam war era. Clinicians routinely use new and effective AEDs with different mechanisms of action to treat patients with epilepsy. If seizures become refractory to medical treatment, clinicians refer the patient to an epilepsy center for surgical evaluation. Specialists admit the patient to an epilepsy monitoring unit (EMU), which is equipped with $24 \mathrm{~h}$ video-EEG telemetry recording to capture seizure events. The patient stays in the EMU from a few days to a couple weeks with the AEDs tapered down or off to capture a sufficient number of seizures. After reviewing the obtained video-EEG data and neuroimaging findings, the epilepsy specialists, including an epileptologist, epilepsy neurosurgeon, neuroradiologist, psychologist, social worker, and nursing coordinator, determine the proper course of treatment in an epilepsy conference. The patient might receive resection surgery to remove the anterior temporal lobe on the nondominant side of the brain or might need further invasive workup with depth/grid electrodes (electrocorticography study).

Specialists consider resection surgery to remove the seizure focus to be the standard of care in patients with epilepsy that is refractory to medical treatment alone. They can also recommend deep brain stimulation or vagus nerve stimulation to improve seizure control in those individuals who have medically refractory epilepsy but for whom resection surgery is not feasible, such as in those patients who have multiple epileptic foci, no identifiable epileptic focus, or an epileptic focus that is not amenable to surgical resection. In some rare instances, specialists can use surgical interruption of the corpus callosum to prevent the abnormal electrical activity spreading to produce a generalized seizure. People with PTE could have multiple cortical epileptic foci, and the techniques used to identify epileptic foci and map eloquent brain areas (which cannot be removed without causing serious neurological deficits) have become quite sophisticated but are not widely available in the VA healthcare system. The recently established VA Epilepsy Centers of Excellence are expected to provide comprehensive epilepsy care in the VA.

\section{Prevention}

Results of Observational Studies for Prophylaxis

A number of published uncontrolled, unblinded observational studies have investigated the effect of 
AEDs in preventing PTE. The AEDs used are phenobarbital (PB) + PHT [11], PHT [12-13], PB [14], and valproic acid (VPA) [15]. Each study included 62 to 390 patients with follow-up periods from 6 months to 13 years. These studies all showed a trend toward a reduced probability of seizures in the treated groups, in which seizures developed in 0 to 10 percent of patients compared with 2 to 50 percent in untreated groups. The only combination treatment, PB + PHT, showed a marked difference between the treated (2\%) and untreated (25\%) groups [11].

\section{Results of Randomized Clinical Trials for Prophylaxis}

Except for one study, researchers did not reproduce the beneficial effects of prophylactic treatment with AEDs in several randomized trials using PHT, PHT + PB, or carbamazepine (CBZ) with follow-up periods of 3 months to 5 years [2]. The only study that showed a prophylactic effect of PHT in preventing PTE was not blinded [2]. Paradoxically, three studies showed a higher incidence of PTE in patients receiving PHT or PB than in untreated groups [16-18].

A Cochrane review using meta-analysis pooled the data from 10 randomized controlled clinical trials with a total of 2,036 patients [8]. It noted that the prophylactic treatment with PHT or CBZ was only effective in reducing the risk of early provoked seizures after TBI. It also noted no beneficial effect in the prevention of PTE with AED treatment. The treated groups seemed to suffer from a higher incidence of AED side effects.

\section{Role of AEDs After Traumatic Brain Injury}

Based on these results, AED prophylaxis seems to be effective in controlling the early provoked seizures, but not in preventing the late unprovoked seizures and PTE. The treatment group suffered from a higher incidence of side effects. Hence, the prophylactic use of AEDs should be limited to the early post-TBI period to prevent provoked seizures. The results to date do not support prophylactic use of AEDs in patients with TBI before the development of PTE. However, researchers have not evaluated any of the newer generation AEDs with different mechanisms of action for PTE prophylaxis. Future studies are needed to clarify this issue.

\section{Potential Pitfalls of Using or Not Using AED Prophylaxis After Traumatic Brain Injury}

Several limiting factors inherent in the clinical trials exist, and caution should be used in interpreting the results. One pitfall is that AEDs tend to have a high incidence of side effects. Many of these side effects are due to actions on the central nervous system, such as cognitive impairment and neurobehavioral problems. These unwanted sides effects could be augmented in patients with TBI. In addition, several lines of evidence from animal studies suggest that AEDs might interfere with the recovery of brain function after TBI [19]. However, in clinical study, it is difficult to tease out AED cognitive side effects from the direct effects of TBI and from AED inhibition of recovery of brain function after TBI [17].

Another limiting factor is the heterogeneity of patient populations. For instance, it is difficult to match both the severity and locations of TBI between placebo and treatment groups. As mentioned earlier in this article, TBI severity positively correlates with the risk of developing PTE. Also, based on animal studies, epilepsy is regionally selective and more likely to occur after lesions in the hippocampus, for example, than after lesions in the cerebellum. The difficulty in controlling the heterogeneity of the TBI patients in clinical trials might obscure the prophylactic effect of AEDs in a specific subgroup of patients with TBI.

The timing of AED treatment after TBI also varies among studies. The timing of AED treatment might be crucial because in one uncontrolled study, researchers gave $1 \mathrm{~g}$ of PHT during the initial resuscitation in the emergency room, which resulted in a marked difference in the risk of PTE; i.e., 10 percent in the treated versus 50 percent in the untreated group [12].

The different types of seizures further complicate the heterogeneity issue. It is still conceivable that a certain subgroup of patients with TBI or certain types of seizures might respond favorably to prophylactic AED treatment. Conversely, nonepileptic seizures might be misdiagnosed as PTE and the patient could be unnecessarily treated with AED.

\section{Epileptogenesis}

\section{Basic Aspects}

Epileptogenesis describes the molecular, cellular, and network processes involved in the development of epilepsy. Although still far from fully understood, many new understandings of the basic mechanisms of epileptogenesis have emerged based on research using various results from animal models of epilepsy. Clinicians commonly observe hyperexcitability in epileptogenesis. They have 
observed the graded increase of hyperexcitability in the kindling models of epilepsy, in which periodic subconvulsive electrical stimulation, for instance, of the amygdala, can induce the sequential and stepwise development of local hyperexcitability, electrographic seizures and, finally, full-blown generalized behavior seizures. Abundant evidence exists for both anatomical and physiological changes as the basis of developing hyperexcitability. For instance, clinicians have described mossy fiber sprouting associated with hippocampal epilepsy after pilocarpine-induced status epilepticus. Physiologically, changes in ion channel kinetics, expressions, numbers of gamma-aminobutyric acid (GABA) and N-methyl-Daspartate receptors, etc., are well described to associate with the development of hyperexcitability and epileptogenesis. Researchers are currently intensely investigating the GABAergic circuit because several lines of evidence show that inhibition of the GABAergic system, or transformation of the GABAergic system to become excitatory, is one of the primary reasons that the neuronal network becomes hyperexcitable. An injury to the superficial layers of GABAergic neurons in TBI could tilt the dedicate balance between the excitatory and inhibitory neurons toward hyperexcitability.

One other well-known observation is that once the kindling process is fully established, it tends to be permanent. This permanence is likely due to certain irreversible changes in the anatomical structures and physiological functions from molecular to network levels.

Agents that inhibit or reverse epileptogenesis are called antiepileptogenic agents. For instance, animal studies have shown GABAergic drugs or VPA to have antiepileptogenic properties. In contrast, drugs that reduce hyperexcitability are called anticonvulsants and could be used to control seizures, either by seizure prevention or abortion. Most of the AEDs on the market are, in fact, anticonvulsants, not antiepileptogenic agents.

\section{Onset}

In theory, one critical issue in PTE prophylaxis is initiation of antiepileptogenic therapy before the epileptogenesis process starts, or at least before it escalates to an irreversible stage. The exact timing of onset of epileptogenesis is unknown, but it could occur very early, even as early as the onset of TBI. This view is supported by the observation that the so-called "latent period," which is the period after the injury but before the onset of first unprovoked seizure, is not a silent period in the pilo- carpine animal epilepsy model. In principle, TBI could set the process of epileptogenesis in motion, and many other accessory factors, such as genetic propensity, neurogenesis, or repair of the brain tissues, will also determine whether epileptogenesis could complete its course of developing PTE.

If this theory of epileptogenesis is correct, the timing of antiepileptic treatment initiation is crucial and should optimally be started right after TBI or even before an expected TBI.

\section{Genetic Propensity for Posttraumatic Epilepsy}

Genetic defects have been identified in several types of epilepsies, mostly the primary generalized epilepsies such as juvenile myoclonic epilepsy or nocturnal frontal lobe epilepsy. However, animal data from transgenic animals and human studies show genotype/phenotype discordance, suggesting that genotype is not the only determining factor for developing epilepsy. These specific genetic defects could be viewed as genetic propensity for developing the phenotype (the epilepsy) when the other cofactors are present.

Given the heterogeneity in PTE described earlier, genetic susceptibility might play a crucial role in developing PTE. However, this role is poorly understood at this point. The Vietnam Head Injury Study showed that a family history of epilepsy was not a significant risk factor for PTE. We could view this finding as lack of positive evidence in humans for a genetic propensity to develop PTE but not as negative evidence against it. In addition, in a recent cohort study of 106 patients with TBI, researchers found that the relative risk of developing PTE for patients with the apolipoprotein E (APOE) 4 allele was 2.41 [20]. The increased risk appears to be independent of the APOE 4 allele's effect on the functional outcome. However, the role of the APOE 4 allele in epileptogenesis is unclear.

\section{Potential New Approaches}

Clinicians have routinely used many newer AEDs that the U.S. Food and Drug Administration approved after the 1990s for the medical management of epilepsies. However, researchers have not systemically investigated these newer AEDs for specific applications in the prevention and treatment of PTE. These newer agents include gabapentin, felbamate, oxcarbazepine, topiramate, tiagabine, vigabatrin, levetiracetam, lamotrigine, pregabalin, zonisamide, rufinamide, and lacosamide. These agents employ different pharmacological mechanisms for seizure control 
than the older AEDs. Animal research has shown some agents to reduce epileptogenesis in kindled epilepsy models [21]. Determining whether these interesting observations in animals apply to PTE prevention in humans requires new translational studies and clinical trials.

\section{PRELIMINARY GUIDELINES FOR DETECTION OF AND THERAPY FOR POSTTRAUMATIC EPILEPSY}

General guidelines for the detection, diagnosis, and treatment of PTE would seem desirable, especially because some types of epilepsy may coexist with and be difficult to distinguish from PTSD. Such guidelines should study-

1. Whether diagnostic screening for TBI is adequate to detect PTE.

2. Whether mild, moderate, or severe TBIs should undergo diagnostic studies to detect PTE (e.g., penetrating head injuries, which have an incidence of PTE $>50 \%$, indicate a reasonable need for EEG and other diagnostic studies; however, we do not have reliable statistics of PTE incidence for blast injuries).

3. Indications for pharmacotherapy and criteria for defining pharmacoresistance and treatment failure.

4. Criteria for surgical evaluation.

5. Surgical indications.

These guidelines would be useful with OIF/OEF veterans to account for comorbidities and other features unique to the veteran population. They would greatly help maintain high-quality care when veterans transfer from the DOD to the VHA or from one VA medical center to another.

\section{Early Treatment}

AED prophylaxis seems to be effective in controlling the early provoked seizures, so early treatment with AEDs up to 1 week after TBI seems advisable. AED treatment should be initiated as early as possible. Longterm AED treatment is not recommended after TBI because no evidence exists from controlled clinical trials that it prevents late unprovoked seizures and the therapy will most likely induce side effects such as cognitive impairment or neurobehavioral problems. Furthermore, we have not completely ruled out the possibility that some AEDs might have a negative effect on the recovery of brain function after TBI.

\section{Indications for Diagnostic Studies}

For early seizure detection after TBI, based on the findings from the epidemiological studies, these patients should optimally receive EEG screening during the first 2 years. EEG might detect interictal epileptiform discharges or electrographic seizures before clinical seizures develop. It is unclear whether the patients with only electrographic seizures or interictal discharges without clinical manifestations should receive AED therapy. If AED therapy is initiated, it is also unclear what clinical parameters clinicians could use to guide the continuation or termination of therapy. However, patients with electrographic seizures or prolonged and active interictal discharges might exhibit subtle clinical signs, such as intermittent spacing out or impaired memory. If these subtle clinical presentations correlate with the epileptiform discharges on video-EEG recordings, we advise initiation of AED therapy by a neurologist.

\section{Pharmacotherapy}

If clinicians discover PTE in a patient, they should initiate AED therapy following the general guidelines used for other types of acquired epilepsy. Monotherapy or, if clinically indicated following failure of one or two single AEDs, polytherapy with a combination of at least two AEDs of different pharmacological mechanisms should be used. Clinicians should follow the general treatment principles of aiming for complete freedom from seizures and using the least amount of AED to achieve satisfactory seizure control with minimal or no side effects.

\section{Indications for Surgery}

Medically refractory cases should receive epilepsy surgery evaluation, which should include inpatient videoEEG monitoring, PET scan, and brain MRI with epilepsy protocols. Some patients will need further evaluations with depth/grid intracranial electrodes, functional MRI, or cortical functional mapping with electrocorticography or other techniques. Clinicians should perform the Wada test for surgical candidates. They should evaluate patients who are refractory but fail surgical evaluation for placement of a deep brain stimulator or vagus nerve stimulator. They should also consider patients who fail vagus nerve stimulation and deep brain stimulation for enrollment in investigational procedures. 


\section{CONCLUSIONS}

PTE might represent a large, complex problem that should not be ignored. Any plans to address the problem should be coordinated with existing polytrauma and TBI programs, and experts are available within the VA to evaluate the problem and design efficient, proactive solutions.

\section{ACKNOWLEDGMENTS}

\author{
Author Contributions: \\ Study concept and design: J. W. Y. Chen, R. L. Ruff, R. Eavey, \\ C. G. Wasterlain. \\ Acquisition of data: J. W. Y. Chen, R. L. Ruff, R. Eavey, \\ C. G. Wasterlain. \\ Analysis and interpretation of data: J. W. Y. Chen. \\ Drafting of manuscript: J. W. Y. Chen, R. L. Ruff, R. Eavey, \\ C. G. Wasterlain. \\ Critical revision of manuscript for important intellectual content: \\ J. W. Y. Chen, R. L. Ruff. \\ Statistical analysis: J. W. Y. Chen, R. L. Ruff. \\ Administrative, technical, or material support: J. W. Y. Chen, \\ C. G. Wasterlain. \\ Study supervision: J. W. Y. Chen, C. G. Wasterlain. \\ Financial Disclosures: The authors have declared that no competing \\ interests exist. \\ Funding/Support: This material was unfunded at the time of manu- \\ script preparation.
}

\section{REFERENCES}

1. Haltiner AM, Temkin NR, Dikmen SS. Risk of seizure recurrence after the first late posttraumatic seizure. Arch Phys Med Rehabil. 1997;78(8):835-40. [PMID: 9344302] DOI:10.1016/S0003-9993(97)90196-9

2. Annegers JF, Hauser WA, Coan SP, Rocca WA. A population-based study of seizures after traumatic brain injuries. N Engl J Med. 1998;338(1):20-24. [PMID: 9414327] DOI:10.1056/NEJM199801013380104

3. Yablon SA. Posttraumatic seizures. Arch Phys Med Rehabil. 1993;74(9):983-1001. [PMID: 8379848]

4. Salazar AM, Jabbari B, Vance SC, Grafman J, Amin D, Dillon JD. Epilepsy after penetrating head injury. I. Clinical correlates: A report of the Vietnam Head Injury Study. Neurology. 1985;35(10):1406-14. [PMID: 3929158$]$

5. Caveness WF, Walker AE, Ascroft PB. Incidence of posttraumatic epilepsy in Korean veterans as compared with those from World War I and World War II. J Neurosurg. 1962;19:122-29. [PMID: 13877492]

DOI:10.3171/jns.1962.19.2.0122
6. Caveness WF. Epilepsy, a product of trauma in our time. Epilepsia. 1976;17(2):207-15. [PMID: 820549$]$

DOI:10.1111/j.1528-1157.1976.tb03398.x

7. Jennett B. Posttraumatic epilepsy. Adv Neurol. 1979;22: 137-47. [PMID: 114000]

8. Schierhout G, Roberts I. Prophylactic antiepileptic agents after head injury: A systematic review. J Neurol Neurosurg Psychiatry. 1998;64(1):108-12. [PMID: 9436738]

9. Taylor DC, Marsh SM. Hughlings Jackson's Dr Z: The paradigm of temporal lobe epilepsy revealed. J Neurol Neurosurg Psychiatry. 1980;43(9):758-67. [PMID: 6999129] DOI:10.1136/jnnp.43.9.758

10. Tanielian T, Jaycox LH, editors. Invisible wounds of war: Psychological and cognitive injuries, their consequences, and services to assist. Santa Monica (CA): RAND Corporation, Center for Military Health Policy Research; 2008.

11. Servit Z, Musil F. Prophylactic treatment of posttraumatic epilepsy: Results of a long-term follow-up in Czechoslovakia. Epilepsia. 1981;22(3):315-20. [PMID: 6786866] DOI:10.1111/j.1528-1157.1981.tb04115.x

12. Wohns RN, Wyler AR. Prophylactic phenytoin in severe head injuries. J Neurosurg. 1979;51(4):507-9.

[PMID: 113510] DOI:10.3171/jns.1979.51.4.0507

13. Young B, Rapp R, Brooks WH, Madauss W, Norton JA. Posttraumatic epilepsy prophylaxis. Epilepsia. 1979;20(6): 671-81. [PMID: 115679] DOI:10.1111/j.1528-1157.1979.tb04851.x

14. Murri L, Arrigo A, Bonuccelli U, Rossi G, Parenti G. Phenobarbital in the prophylaxis of late posttraumatic seizures. Ital J Neurol Sci. 1992;13(9):755-60. [PMID: 1483858] DOI:10.1007/BF02229161

15. Price D. The efficacy of sodium valproate as the only anticonvulsant administrated to neurosurgical patients. In: Parsonage MJ, Caldwell AD, editors. The place of sodium valproate in the treatment of epilepsy. London (England): Royal Society of Medicine; 1980. p. 23-34.

16. Young B, Rapp RP, Norton JA, Haack D, Tibbs PA, Bean JR. Failure of prophylactically administered phenytoin to prevent late posttraumatic seizures. J Neurosurg. 1983;58(2): 236-41. [PMID: 6848681]

DOI:10.3171/jns.1983.58.2.0236

17. Temkin NR, Dikmen SS, Wilensky AJ, Keihm J, Chabal S, Winn HR. A randomized, double-blind study of phenytoin for the prevention of post-traumatic seizures. $\mathrm{N}$ Engl $\mathrm{J}$ Med. 1990;323(8):497-502. [PMID: 2115976]

18. Manaka S. Cooperative prospective study on posttraumatic epilepsy: Risk factors and the effect of prophylactic anticonvulsant. Jpn J Psychiatry Neurol. 1992;46(2):311-15. [PMID: 1434154]

19. Hernandez TD. Preventing post-traumatic epilepsy after brain injury: Weighing the costs and benefits of anticonvulsant 
prophylaxis. Trends Pharmacol Sci. 1997;18(2):59-62.

[PMID: 9090312]

DOI:10.1016/S0165-6147(97)89801-X

20. Diaz-Arrastia R, Gong Y, Fair S, Scott KD, Garcia MC, Carlile MC, Agostini MA, Van Ness PC. Increased risk of late posttraumatic seizures associated with inheritance of APOE epsilon4 allele. Arch Neurol. 2003;60(6):818-22. [PMID: 12810485]

DOI:10.1001/archneur.60.6.818
21. Temkin NR, Jarell AD, Anderson GD. Antiepileptogenic agents: How close are we? Drugs. 2001;61(8):1045-55.

[PMID: 11465868]

DOI:10.2165/00003495-200161080-00002

Submitted for publication September 23, 2008. Accepted in revised form July 22, 2009. 